\title{
A CONVEX COMBINATION OF TWO-SAMPLE U-STATISTICS
}

\author{
Koichiro Toda* and Hajime Yamato**
}

\begin{abstract}
A convex combination of one-sample U-statistics was introduced by Toda and Yamato (2001) and its Edgeworth expansion was derived by Yamato et al. (2003). We introduce a convex combination of two-sample U-statistics, which includes twosample U-statistic, V-statistic and limit of Bayes estimate. Its Edgeworth expansion is derived with remainder term $o\left(N^{-1 / 2}\right)$, under the condition that the kernel is nondegenerate. We give some examples of the expansion for three statistics, two-sample U-statistic, V-statistic and limit of Bayes estimate, based on some distributions.
\end{abstract}

Key words and phrases: Convex combination, two-sample U-statistic, two-sample V-statistic.

\section{Introduction}

Let $F$ and $G$ be continuous distributions on the real line. Let $\theta=\theta(F, G)$ be a regular functional of $F$ and $G$. That is, there exists a measurable function $h\left(x_{1}, \ldots, x_{k_{1}} ; y_{1}, \ldots, y_{k_{2}}\right)$ such that

$$
\theta=\int_{-\infty}^{\infty} \int_{-\infty}^{\infty} h\left(x_{1}, \ldots, x_{k_{1}} ; y_{1}, \ldots, y_{k_{2}}\right) \prod_{i=1}^{k_{1}} d F\left(x_{i}\right) \prod_{j=1}^{k_{2}} d G\left(y_{j}\right)
$$

We assume that $h\left(x_{1}, \ldots, x_{k_{1}} ; y_{1}, \ldots, y_{k_{2}}\right)$ is symmetric with respect to $x_{1}, \ldots, x_{k_{1}}$ and $y_{1}, \ldots, y_{k_{2}}$, respectively, and the integers $k_{1}$ and $k_{2}$ are the smallest integers satisfying (1.1). The function $h$ is called the kernel of $\theta$ and $\left(k_{1}, k_{2}\right)$ is called the degree of $h$ and/or $\theta$.

Let $X_{1}, \ldots, X_{n_{1}}$ and $Y_{1}, \ldots, Y_{n_{2}}$ be two independent samples of sizes $n_{1}$ and $n_{2}$ from the distributions $F$ and $G$, respectively. As estimators of $\theta$, two-sample U-statistic and V-statistic are well-known. The two-sample U-statistic $U_{n_{1}, n_{2}}$ is given by

$$
U_{n_{1}, n_{2}}=\left(\begin{array}{l}
n_{1} \\
k_{1}
\end{array}\right)^{-1}\left(\begin{array}{l}
n_{2} \\
k_{2}
\end{array}\right)^{-1} \sum_{\left(n_{1}, k_{1}\right)} \sum_{\left(n_{2}, k_{2}\right)} h\left(X_{i_{1}}, \ldots, X_{i_{k_{1}}} ; Y_{j_{1}}, \ldots, Y_{j_{k_{2}}}\right),
$$

where the summation $\sum_{\left(n_{1}, k_{1}\right)}$ is taken over all possible $i_{1}, \ldots, i_{k_{1}}$ satisfying $1 \leq i_{1}<\cdots<i_{k_{1}} \leq n_{1}$. The two-sample V-statistic $V_{n_{1}, n_{2}}$ is given by

$$
V_{n_{1}, n_{2}}=n_{1}^{-k_{1}} n_{2}^{-k_{2}}
$$

Received March 4, 2005. Revised June 28, 2005. Accepted November 11, 2005.

*Kagoshima Koto Preparatory School, Kagoshima 890-0051, Japan.

**Department of Mathematics and Computer Science, Kagoshima University, Kagoshima 890-0065, Japan. 


$$
\times \sum_{i_{1}=1}^{n_{1}} \cdots \sum_{i_{k_{1}}=1}^{n_{1}} \sum_{j_{1}=1}^{n_{2}} \cdots \sum_{j_{k_{2}}=1}^{n_{2}} h\left(X_{i_{1}}, \ldots, X_{i_{k_{1}}} ; Y_{j_{1}}, \ldots, Y_{j_{k_{2}}}\right) \text {. }
$$

The followings are examples of $\theta(F, G)$ : (i) The kernel $h(x, y)=x-y$ gives the parameter $\theta=E\left(X_{1}\right)-E\left(Y_{1}\right)$. (ii) The kernel $h(x, y)=1(x \leq y)$ and $=0$ $(x>y)$ gives the parameter $\theta=P\left(X_{1} \leq Y_{1}\right)$. The corresponding U-statistic is related to the two-sample Wilcoxon (Mann-Whitney) rank sum statistic $W$ by the relation $W=n_{1} n_{2} U_{n_{1}, n_{2}}+n_{2}\left(n_{2}+1\right) / 2$. (iii) The kernel $h\left(x_{1}, x_{2} ; y_{1}, y_{2}\right)=1 / 3$ $\left(x_{1}, x_{2}<y_{1}, y_{2}\right.$ or $\left.y_{1}, y_{2}<x_{1}, x_{2}\right)$ and $=-1 / 6$ (otherwise) gives the parameter $\Delta=\int_{-\infty}^{\infty}[F(x)-G(x)]^{2} d([F(x)+G(x)] / 2)$, which is regarded as a distance between the two distributions $F$ and $G$. (iv) The kernel $h\left(x_{1}, x_{2} ; y_{1}, y_{2}\right)=1$ $\left(\left|y_{1}-y_{2}\right|>\left|x_{1}-x_{2}\right|\right)$ and $=0$ (otherwise) gives the parameter $\theta=P\left(\left|Y_{1}-Y_{2}\right|>\right.$ $\left.\left|X_{1}-X_{2}\right|\right)$. The associated U-statistic appears in the testing problem of twosample scale by Lehmann (1951). (v) The kernel $h\left(x_{1}, x_{2} ; y_{1}, y_{2}\right)=1\left(x_{1}+x_{2}<\right.$ $\left.y_{1}+y_{2}\right)$ and $=0$ (otherwise) gives the parameter $\theta=P\left(X_{1}+X_{2}<Y_{1}+Y_{2}\right.$ ), which is a measure of the difference in location considered by Hollander (1967). (See, for example, Koroljuk and Borovskich (1994), and Randles and Wolfe (1979).)

Yamato (1977) derives the Bayes estimate of $\theta$ using Dirichlet prior process of Ferguson (1973), and gives the limit of Bayes estimate which is given by

$$
\begin{aligned}
B_{n_{1}, n_{2}}= & \left(\begin{array}{c}
n_{1}+k_{1}-1 \\
k_{1}
\end{array}\right)^{-1}\left(\begin{array}{c}
n_{2}+k_{2}-1 \\
k_{2}
\end{array}\right)^{-1} \sum_{r_{1}} \sum_{r_{1}+\cdots+r_{n_{1}}=k_{1}} \sum_{s_{1}+\cdots+s_{n_{2}}=k_{2}} \\
& h(\underbrace{X_{1}^{X_{1}, \ldots, X_{1}}}_{s_{1}}, \ldots, \underbrace{X_{n_{1}}, \ldots, X_{n_{1}}}_{r_{n_{1}}} ;
\end{aligned}
$$

where the summation $\sum_{r_{1}+\cdots+r_{n_{1}}=k_{1}}$ is taken over all nonnegative integers $r_{1}, \ldots, r_{n_{1}}$ satisfying $r_{1}+\cdots+r_{n_{1}}=k_{1}$. This statistic is abbreviated to LBstatistic.

All the above two-sample statistics $U_{n_{1}, n_{2}}, V_{n_{1}, n_{2}}$ and $B_{n_{1}, n_{2}}$ can be denoted by a convex combination of two-sample U-statistics. This convex combination $Y_{n_{1}, n_{2}}$ is introduced in Section 2. In this paper, we put $N=n_{1}+n_{2}$ and consider the asymptotic properties of statistics under the condition such that

$$
\frac{n_{1}}{N} \rightarrow p, \quad \frac{n_{2}}{N} \rightarrow 1-p \quad \text { as } \quad N \rightarrow \infty
$$

where $0<p<1$ is a constant. In Section 3, we give an asymptotic expansion of $Y_{n_{1}, n_{2}}$ as $N$ tends to $\infty$.

The two-sample U-statistic $U_{n_{1}, n_{2}}$ has asymptotic normality (see, for example, Koroljuk and Borovskich (1994), Lee (1990), and Randles and Wolfe (1979)). From this, it is shown that the two-sample Y-statistic $Y_{n_{1}, n_{2}}$ has also the same asymptotic normality. To see the difference between asymptotic distributions of 
these two statistics, we need their Edgeworth expansion. The Edgeworth expansion of the two-sample U-statistic $U_{n_{1}, n_{2}}$ was derived by Koroljuk and Borovskich (1994), and Maesono (1985). We shall derive the Edgeworth expansion of $Y_{n_{1}, n_{2}}$ in Section 4.

For the same parameter $\theta$, Edgeworth expansion of $Y_{n_{1}, n_{2}}$ depends on the weight function $w$. In Section 5 , for 4 kernels we give examples of the expansion for the statistics $V_{n_{1}, n_{2}}, S_{n_{1}, n_{2}}$ and $B_{n_{1}, n_{2}}$, based on some special distributions.

\section{A convex combination of two-sample U-statistics}

Let $w\left(\alpha_{1}, \ldots, \alpha_{j} ; k\right)$ be a nonnegative and symmetric function of positive integers $\alpha_{1}, \ldots, \alpha_{j}$ such that $j=1, \ldots, k$ and $\alpha_{1}+\cdots+\alpha_{j}=k$ for a given integer $k$. We assume that at least one of $w\left(\alpha_{1}, \ldots, \alpha_{j} ; k\right)$ 's is positive. We put

$$
d(k, j)=\sum_{\alpha_{1}+\cdots+\alpha_{j}=k}^{+} w\left(\alpha_{1}, \ldots, \alpha_{j} ; k\right), \quad j=1,2, \ldots, k,
$$

where the summation $\sum_{\alpha_{1}+\cdots+\alpha_{j}=k}^{+}$is taken over all positive integers $\alpha_{1}, \ldots, \alpha_{j}$ satisfying $\alpha_{1}+\cdots+\alpha_{j}=k$ for $j$ and $k$ given.

For $j_{1}=1, \ldots, k_{1}$ and $j_{2}=1, \ldots, k_{2}$, let $h_{\left(j_{1}, j_{2}\right)}\left(x_{1}, \ldots, x_{j_{1}} ; y_{1}, \ldots, y_{j_{2}}\right)$ be the kernel given by

$$
\begin{aligned}
h_{\left(j_{1}, j_{2}\right)} & \left(x_{1}, \ldots, x_{j_{1}} ; y_{1}, \ldots, y_{j_{2}}\right) \\
= & \frac{1}{d\left(k_{1}, j_{1}\right) d\left(k_{2}, j_{2}\right)} \sum_{r_{1}+\cdots+r_{j_{1}}=k_{1}}^{+} \sum_{s_{1}+\cdots+s_{j_{2}}=k_{2}}^{+} \\
& w\left(r_{1}, \ldots, r_{j_{1}} ; k_{1}\right) w\left(s_{1}, \ldots, s_{j_{2}} ; k_{2}\right) \\
& \times h(\underbrace{x_{1}, \ldots, x_{1}}_{r_{1}}, \ldots, \underbrace{x_{j_{1}}, \ldots, x_{j_{1}}}_{r_{j_{1}}} ; \underbrace{y_{1}, \ldots, y_{1}}_{s_{1}}, \ldots, \underbrace{y_{j_{2}}, \ldots, y_{j_{2}}}_{s_{j_{2}}}) .
\end{aligned}
$$

Let $U_{n_{1}, n_{2}}^{\left(j_{1}, j_{2}\right)}$ be the two-sample U-statistic associated with this kernel $h_{\left(j_{1}, j_{2}\right)}$ for $j_{1}=1, \ldots, k_{1}$ and $j_{2}=1, \ldots, k_{2}$.

The kernel $h_{\left(j_{1}, j_{2}\right)}\left(x_{1}, \ldots, x_{j_{1}} ; y_{1}, \ldots, y_{j_{2}}\right)$ is symmetric with respect to $x_{1}, \ldots, x_{j_{1}}$ and $y_{1}, \ldots, y_{j_{2}}$, respectively, because of the symmetry of $w\left(\alpha_{1}, \ldots\right.$, $\left.\alpha_{j} ; k\right)$. If $d\left(k_{1}, j_{1}\right)$ or $d\left(k_{2}, j_{2}\right)$ equal zero for some $j_{1}$ or $j_{2}$, respectively, then the associated $w\left(\alpha_{1}, \ldots, \alpha_{j_{1}} ; k_{1}\right)$ 's or $w\left(\alpha_{1}, \ldots, \alpha_{j_{2}} ; k_{2}\right)$ 's are equal to zero. In this case, we let the corresponding statistic $U_{n_{1}, n_{2}}^{\left(j_{1}, j_{2}\right)}$ to be zero. Especially, if $w(1, \ldots, 1 ; k)>0$ then we have

$$
h_{\left(k_{1}, k_{2}\right)}=h \quad \text { and } \quad U_{n_{1}, n_{2}}^{\left(k_{1}, k_{2}\right)}=U_{n_{1}, n_{2}},
$$

because of $d\left(k_{1}, k_{1}\right)=w\left(1, \ldots, 1 ; k_{1}\right)$ and $d\left(k_{2}, k_{2}\right)=w\left(1, \ldots, 1 ; k_{2}\right)$. If $w(1, \ldots, 1 ; k)>0$ and $w(1, \ldots, 1,2 ; k)>0$, then

$$
\begin{aligned}
& h_{\left(k_{1}-1, k_{2}\right)}\left(x_{1}, x_{2}, \ldots, x_{k_{1}-1} ; y_{1}, \ldots, y_{k_{2}}\right) \\
&=\frac{1}{k_{1}-1}[ h\left(x_{1}, x_{1}, x_{2}, \ldots, x_{k_{1}-1} ; y_{1}, \ldots, y_{k_{2}}\right) \\
&+h\left(x_{1}, x_{2}, x_{2}, x_{3}, \ldots, x_{k_{1}-1} ; y_{1}, \ldots, y_{k_{2}}\right) \\
&\left.+\cdots+h\left(x_{1}, x_{2}, \ldots, x_{k_{1}-2}, x_{k_{1}-1}, x_{k_{1}-1} ; y_{1}, \ldots, y_{k_{2}}\right)\right]
\end{aligned}
$$


and

$$
\begin{aligned}
& h_{\left(k_{1}, k_{2}-1\right)}\left(x_{1}, \ldots, x_{k_{1}} ; y_{1}, \ldots, y_{k_{2}-1}\right) \\
&=\frac{1}{k_{2}-1}[ h\left(x_{1}, \ldots, x_{k_{1}} ; y_{1}, y_{1}, y_{2}, \ldots, y_{k_{2}-1}\right) \\
&+h\left(x_{1}, \ldots, x_{k_{1}} ; y_{1}, y_{2}, y_{2}, y_{3}, \ldots, y_{k_{2}-1}\right) \\
&\left.+\cdots+h\left(x_{1}, \ldots, x_{k_{1}} ; y_{1}, \ldots, y_{k_{2}-2}, y_{k_{2}-1}, y_{k_{2}-1}\right)\right] .
\end{aligned}
$$

Definition 2.1. As an estimator of $\theta$, a convex combination of twosample U-statistics is defined by

$$
Y_{n_{1}, n_{2}}=\frac{1}{D\left(n_{1}, k_{1}\right) D\left(n_{2}, k_{2}\right)} \sum_{j_{1}=1}^{k_{1}} \sum_{j_{2}=1}^{k_{2}} d\left(k_{1}, j_{1}\right) d\left(k_{2}, j_{2}\right)\left(\begin{array}{c}
n_{1} \\
j_{1}
\end{array}\right)\left(\begin{array}{c}
n_{2} \\
j_{2}
\end{array}\right) U_{n_{1}, n_{2}}^{\left(j_{1}, j_{2}\right)}
$$

where $D(n, k)=\sum_{j=1}^{k} d(k, j)\left(\begin{array}{c}n \\ j\end{array}\right)$.

Since $w$ 's are nonnegative and at least one of them is positive, $D(n, k)$ is positive.

If we choose the weight function $w$, the statistic $Y_{n_{1}, n_{2}}$ is determined as an estimator of $\theta$. For example, let $w$ be the function given by

$$
w(1,1, \ldots, 1 ; k)=1 \quad \text { and } \quad w\left(\alpha_{1}, \ldots, \alpha_{j} ; k\right)=0 \quad \text { for } \quad j=1, \ldots, k-1 .
$$

Then we have $d(k, k)=1, d(k, j)=0(j=1, \ldots, k-1)$ and $D(n, k)=\left(\begin{array}{l}n \\ k\end{array}\right)$. Thus $Y_{n_{1}, n_{2}}$ is equal to the two-sample U-statistic $U_{n_{1}, n_{2}}$.

Let $w$ be the function given by

$$
w\left(\alpha_{1}, \ldots, \alpha_{j} ; k\right)=\frac{k !}{\alpha_{1} ! \cdots \alpha_{j} !}
$$

for positive integers $\alpha_{1}, \ldots, \alpha_{j}$ such that $j=1, \ldots, k$ and $\alpha_{1}+\cdots+\alpha_{j}=k$. Then it holds that $\sum_{\alpha_{1}+\cdots+\alpha_{j}=k}^{+} w\left(\alpha_{1}, \ldots, \alpha_{j} ; k\right)=j ! \mathcal{S}(k, j)$, where $\mathcal{S}(k, j)$ is the Stirling number of the second kind. (For the Stirling numbers, see for example, Charalambides and Singh (1988).) Hence, we have $d(k, j)=j ! \mathcal{S}(k, j)$ for $j=$ $1, \ldots, k$ and $D(n, k)=\sum_{j=1}^{k} \mathcal{S}(k, j)(n)_{j}=n^{k}$, where $(n)_{j}=n(n-1) \cdots(n-j+$ $1)$. Thus the kernel $h_{\left(j_{1}, j_{2}\right)}\left(x_{1}, \ldots, x_{j_{1}} ; y_{1}, \ldots, y_{j_{2}}\right)$ is equal to

$$
\begin{array}{r}
\frac{1}{j_{1} ! \mathcal{S}\left(k_{1}, j_{1}\right) j_{2} ! \mathcal{S}\left(k_{2}, j_{2}\right)} \sum_{r_{1}+\cdots+r_{j_{1}}=k_{1}}^{+} \sum_{s_{1}+\cdots+s_{j_{2}}=k_{2}}^{+} \frac{k_{1} !}{r_{1} ! \cdots r_{j_{1}} ! s_{1} ! \cdots s_{j_{2}} !} \frac{k_{2} !}{r_{r_{1}}}, \ldots, \underbrace{x_{j_{1}}, \ldots, x_{j_{1}}}_{r_{j_{1}}} ; \underbrace{y_{1}, \ldots, y_{1}}_{s_{1}}, \ldots, \underbrace{y_{j_{2}}, \ldots, x_{j_{2}}}_{s_{j_{2}}}) .
\end{array}
$$

By the U-statistics $U_{n_{1}, n_{2}}^{\left(j_{1}, j_{2}\right)}$ associated with these kernels, the statistic $Y_{n_{1}, n_{2}}$ is written as

$$
V_{n_{1}, n_{2}}=\frac{1}{n_{1}^{k_{1}} n_{2}^{k_{2}}} \sum_{j_{1}=1}^{k_{1}} \sum_{j_{2}=1}^{k_{2}} \mathcal{S}\left(k_{1}, j_{1}\right) \mathcal{S}\left(k_{2}, j_{2}\right)\left(n_{1}\right)_{j_{1}}\left(n_{2}\right)_{j_{2}} U_{n_{1}, n_{2}}^{\left(j_{1}, j_{2}\right)},
$$


which is equal to the two-sample $\mathrm{V}$-statistic $V_{n_{1}, n_{2}}$ given by (1.3) (see, Toda and Yamato (2001), p. 227-228).

Let $w$ be the function given by

$$
w\left(\alpha_{1}, \ldots, \alpha_{j} ; k\right)=1
$$

for positive integers $\alpha_{1}, \ldots, \alpha_{j}$ such that $j=1, \ldots, k$ and $\alpha_{1}+\cdots+\alpha_{j}=k$. Then, we have $d(k, j)=\left(\begin{array}{c}k-1 \\ j-1\end{array}\right)$ for $j=1, \ldots, k$ and $D(n, k)=\sum_{j=1}^{k}\left(\begin{array}{c}k-1 \\ j-1\end{array}\right)\left(\begin{array}{c}n \\ j\end{array}\right)=\left(\begin{array}{c}n+k-1 \\ k\end{array}\right)$. Thus the kernel $h_{\left(j_{1}, j_{2}\right)}\left(x_{1}, \ldots, x_{j_{1}} ; y_{1}, \ldots, y_{j_{2}}\right)$ is equal to

$$
\begin{aligned}
\left(\begin{array}{c}
k_{1}-1 \\
j_{1}-1
\end{array}\right)^{-1}\left(\begin{array}{c}
k_{2}-1 \\
j_{2}-1
\end{array}\right) \sum_{r_{1}+\cdots+r_{j_{1}}=k_{1}}^{\sum_{s_{1}+\cdots+s_{j_{2}}=k_{2}} \sum_{s_{1}}^{+}} & \underbrace{+}_{r_{1}}, \underbrace{x_{1}, \ldots, x_{1}}_{r_{j_{1}}}, \ldots, \underbrace{x_{j_{1}}, \ldots, x_{j_{1}}}_{s_{j_{2}}}, \ldots, \underbrace{y_{1}, \ldots, y_{1}}_{y_{1}}, \ldots .
\end{aligned}
$$

By the U-statistics $U_{n_{1}, n_{2}}^{\left(j_{1}, j_{2}\right)}$ associated with these kernels, the statistic $Y_{n_{1}, n_{2}}$ is written as

$$
\begin{aligned}
B_{n_{1}, n_{2}}= & \left(\begin{array}{c}
n_{1}+k_{1}-1 \\
k_{1}
\end{array}\right)^{-1}\left(\begin{array}{c}
n_{2}+k_{2}-1 \\
k_{2}
\end{array}\right)^{-1} \\
& \times \sum_{j_{1}=1}^{k_{1}} \sum_{j_{2}=1}^{k_{2}}\left(\begin{array}{c}
k_{1}-1 \\
j_{1}-1
\end{array}\right)\left(\begin{array}{c}
k_{2}-1 \\
j_{2}-1
\end{array}\right)\left(\begin{array}{c}
n_{1} \\
j_{1}
\end{array}\right)\left(\begin{array}{c}
n_{2} \\
j_{2}
\end{array}\right) U_{n_{1}, n_{2}}^{\left(j_{1}, j_{2}\right)},
\end{aligned}
$$

which is equal to $B_{n_{1}, n_{2}}$ given by (1.4) (see, Toda and Yamato (2001), p. 227).

Let $w$ be the function given by

$$
w\left(\alpha_{1}, \ldots, \alpha_{j} ; k\right)=\frac{k !}{\alpha_{1} \cdots \alpha_{j}}
$$

for positive integers $\alpha_{1}, \ldots, \alpha_{j}$ such that $j=1, \ldots, k$ and $\alpha_{1}+\cdots+\alpha_{j}=k$. Then it holds that $\sum_{\alpha_{1}+\cdots+\alpha_{j}=k}^{+} w\left(\alpha_{1}, \ldots, \alpha_{j} ; k\right)=j !|\boldsymbol{s}(k, j)|$, where $\boldsymbol{s}(k, j)$ is the Stirling number of the first kind. Hence, we have $d(k, j)=j !|s(k, j)|$ for $j=1, \ldots, k$ and

$$
D(n, k)=\sum_{j=1}^{k}|\boldsymbol{s}(k, j)|(n)_{j}
$$

Thus the kernel $h_{\left(j_{1}, j_{2}\right)}\left(x_{1}, \ldots, x_{j_{1}} ; y_{1}, \ldots, y_{j_{2}}\right)$ is equal to

$$
\begin{aligned}
& \frac{1}{j_{1} ! j_{2} !\left|\boldsymbol{s}\left(k_{1}, j_{1}\right) \boldsymbol{s}\left(k_{2}, j_{2}\right)\right|} \sum_{r_{1}+\cdots+r_{j_{1}}=k_{1}}^{+} \sum_{s_{1}+\cdots+s_{j_{2}}=k_{2}}^{+} \frac{k_{1} !}{r_{1} \cdots r_{j_{1}}} \frac{k_{2} !}{s_{1} \cdots s_{j_{2}}} \\
& \times h(\underbrace{x_{1}, \ldots, x_{1}}_{r_{1}}, \ldots, \underbrace{x_{j_{1}}, \ldots, x_{j_{1}}}_{r_{j_{1}}} ; \underbrace{y_{1}, \ldots, y_{1}}_{s_{1}}, \ldots, \underbrace{y_{j_{2}}, \ldots, x_{j_{2}}}_{s_{j_{2}}}) .
\end{aligned}
$$

By the U-statistics $U_{n_{1}, n_{2}}^{\left(j_{1}, j_{2}\right)}$ associated with these kernels, the statistic $Y_{n_{1}, n_{2}}$ is written as

$$
S_{n_{1}, n_{2}}=\frac{1}{D\left(n_{1}, k_{1}\right) D\left(n_{2}, k_{2}\right)} \sum_{j_{1}=1}^{k_{1}} \sum_{j_{2}=1}^{k_{2}}\left|\boldsymbol{s}\left(k_{1}, j_{1}\right) \boldsymbol{s}\left(k_{2}, j_{2}\right)\right|\left(n_{1}\right)_{j_{1}}\left(n_{2}\right)_{j_{2}} U_{n_{1}, n_{2}}^{\left(j_{1}, j_{2}\right)},
$$


(compare with Nomachi et al. (2002), p. 97-98).

\section{Asymptotic expansion of $Y$-statistic}

If $k_{1}=1$ and $k_{2}=1$, then the statistic $Y_{n_{1}, n_{2}}$ is equal to $U_{n_{1}, n_{2}}$. Therefore hereafter we assume that $k_{1} k_{2} \geq 2$. We suppose $d(k, k)>0$, which is equivalent to $w(1, \ldots, 1 ; k)>0$. Then, with $\delta_{k}=k d(k, k-1) / d(k, k)$ it holds that

$$
\begin{aligned}
& \frac{d(k, k)}{D(n, k)}\left(\begin{array}{l}
n \\
k
\end{array}\right)=1-\frac{\delta_{k}}{n}+O\left(\frac{1}{n^{2}}\right), \\
& \frac{d(k, k-1)}{D(n, k)}\left(\begin{array}{c}
n \\
k-1
\end{array}\right)=\frac{\delta_{k}}{n}+O\left(\frac{1}{n^{2}}\right) .
\end{aligned}
$$

and

$$
\frac{d(k, j)}{D(n, k)}\left(\begin{array}{l}
n \\
j
\end{array}\right)=O\left(\frac{1}{n^{2}}\right), \quad j=1, \ldots, k-2 .
$$

For the U-statistic $U_{n_{1}, n_{2}}, d(k, k) n^{(k)} /[D(n, k) k !]=1$ and $\delta_{k}=0$. For the Vstatistic $V_{n_{1}, n_{2}}$ and the statistic $S_{n_{1}, n_{2}}, \delta_{k}=k(k-1) / 2$. For the statistic $B_{n_{1}, n_{2}}$, $\delta_{k}=k(k-1)$ (see Nomachi et al. (2002)).

We assume that

$$
\begin{gathered}
E\left\{h(\underbrace{X_{1}^{X_{1}, \ldots, X_{1}}, \ldots, \underbrace{X_{j_{1}}, \ldots, X_{j_{1}}}_{r_{j_{1}}} ; \underbrace{Y_{1}, \ldots, Y_{1}}_{s_{1}}, \ldots, \underbrace{Y_{j_{2}}, \ldots, Y_{j_{2}}}_{s_{j_{2}}})\}^{2}}_{r_{1}}\right. \\
<\infty
\end{gathered}
$$

for positive integers $r_{1}, \ldots, r_{j_{1}}$ and $s_{1}, \ldots, s_{j_{2}}$ satisfying $r_{1}+\cdots+r_{j_{1}}=k_{1}$ $\left(j_{1}=1, \ldots, k_{1}\right)$ and $s_{1}+\cdots+s_{j_{2}}=k_{2}\left(j_{2}=1, \ldots, k_{2}\right)$, respectively. Then there exist $E\left\{U_{n_{1}, n_{2}}^{\left(j_{1}, j_{2}\right)}\right\}^{2}$ for $j_{1}=1, \ldots, k_{1}$ and $j_{2}=1, \ldots, k_{2}$. Thus we have for $j_{1}=1, \ldots, k_{1}$ and $j_{2}=1, \ldots, k_{2}$

$$
E\left\{U_{n_{1}, n_{2}}^{\left(j_{1}, j_{2}\right)}\right\}^{2}<\infty, \quad \text { and } \quad \operatorname{Var}\left(U_{n_{1}, n_{2}}^{\left(j_{1}, j_{2}\right)}\right)=O\left(N^{-1}\right)
$$

Then we have the following asymptotic expansion of the statistic $Y_{n_{1}, n_{2}}$.

Proposition 3.1. Under the conditions $w(1, \ldots, 1 ; k)>0$ and $(3.4)$, we have

$$
Y_{n_{1}, n_{2}}-\theta=\left(U_{n_{1}, n_{2}}-\theta\right)+\frac{1}{N} b^{(0)}+R_{n_{1}, n_{2}}
$$

where $E\left[\left|R_{n_{1}, n_{2}}\right|^{2}\right]=o\left(N^{-2}\right)$,

$$
b^{(0)}=\frac{\delta_{k_{1}}}{p}\left(\theta^{\left(k_{1}-1, k_{2}\right)}-\theta\right)+\frac{\delta_{k_{2}}}{1-p}\left(\theta^{\left(k_{1}, k_{2}-1\right)}-\theta\right)
$$


and

$$
\begin{aligned}
& \theta^{\left(k_{1}-1, k_{2}\right)}=E\left[h\left(X_{1}, X_{1}, X_{2}, X_{3}, \ldots, X_{k_{1}-1} ; Y_{1}, Y_{2}, \ldots, Y_{k_{2}}\right)\right] \\
& \theta^{\left(k_{1}, k_{2}-1\right)}=E\left[h\left(X_{1}, X_{2}, \ldots, X_{k_{1}} ; Y_{1}, Y_{1}, Y_{2}, Y_{3}, \ldots, Y_{k_{2}-1}\right)\right]
\end{aligned}
$$

Proof. We note

$$
E\left[U_{n_{1}, n_{2}}^{\left(k_{1}, k_{2}-1\right)}\right]=\theta^{\left(k_{1}, k_{2}-1\right)} \quad \text { and } \quad E\left[U_{n_{1}, n_{2}}^{\left(k_{1}-1, k_{2}\right)}\right]=\theta^{\left(k_{1}-1, k_{2}\right)}
$$

From (2.5), we can write

$$
Y_{n_{1}, n_{2}}-\theta=I_{n_{1}, n_{2}}^{(1)}+I_{n_{1}, n_{2}}^{(2)}+I_{n_{1}, n_{2}}^{(3)}+b_{n_{1}, n_{2}}+R_{n_{1}, n_{2}}^{*},
$$

where

$$
\begin{aligned}
I_{n_{1}, n_{2}}^{(1)}= & \frac{d\left(k_{1}, k_{1}\right) d\left(k_{2}, k_{2}\right)}{D\left(n_{1}, k_{1}\right) D\left(n_{2}, k_{2}\right)}\left(\begin{array}{l}
n_{1} \\
k_{1}
\end{array}\right)\left(\begin{array}{l}
n_{2} \\
k_{2}
\end{array}\right)\left(U_{n_{1}, n_{2}}-\theta\right), \\
I_{n_{1}, n_{2}}^{(2)}= & \frac{d\left(k_{1}, k_{1}\right) d\left(k_{2}, k_{2}-1\right)}{D\left(n_{1}, k_{1}\right) D\left(n_{2}, k_{2}\right)}\left(\begin{array}{l}
n_{1} \\
k_{1}
\end{array}\right)\left(\begin{array}{c}
n_{2} \\
k_{2}-1
\end{array}\right)\left(U_{n_{1}, n_{2}}^{\left(k_{1}, k_{2}-1\right)}-\theta^{\left(k_{1}, k_{2}-1\right)}\right), \\
I_{n_{1}, n_{2}}^{(3)}= & \frac{d\left(k_{1}, k_{1}-1\right) d\left(k_{2}, k_{2}\right)}{D\left(n_{1}, k_{1}\right) D\left(n_{2}, k_{2}\right)}\left(\begin{array}{c}
n_{1} \\
k_{1}-1
\end{array}\right)\left(\begin{array}{c}
n_{2} \\
k_{2}
\end{array}\right)\left(U_{n_{1}, n_{2}}^{\left(k_{1}-1, k_{2}\right)}-\theta^{\left(k_{1}-1, k_{2}\right)}\right), \\
b_{n_{1}, n_{2}}= & \frac{d\left(k_{1}, k_{1}\right) d\left(k_{2}, k_{2}-1\right)}{D\left(n_{1}, k_{1}\right) D\left(n_{2}, k_{2}\right)}\left(\begin{array}{c}
n_{1} \\
k_{1}
\end{array}\right)\left(\begin{array}{c}
n_{2} \\
k_{2}-1
\end{array}\right)\left(\theta^{\left(k_{1}, k_{2}-1\right)}-\theta\right) \\
& +\frac{d\left(k_{1}, k_{1}-1\right) d\left(k_{2}, k_{2}\right)}{D\left(n_{1}, k_{1}\right) D\left(n_{2}, k_{2}\right)}\left(\begin{array}{c}
n_{1} \\
k_{1}-1
\end{array}\right)\left(\begin{array}{c}
n_{2} \\
k_{2}
\end{array}\right)\left(\theta^{\left(k_{1}-1, k_{2}\right)}-\theta\right),
\end{aligned}
$$

and

$$
\begin{aligned}
R_{n_{1}, n_{2}}^{*}=\frac{1}{D\left(n_{1}, k_{1}\right) D\left(n_{2}, k_{2}\right)}[ & d\left(k_{1}, k_{1}\right)\left(\begin{array}{c}
n_{1} \\
k_{1}
\end{array}\right) \sum_{j_{2}=1}^{k_{2}-2} d\left(k_{2}, j_{2}\right)\left(\begin{array}{c}
n_{2} \\
j_{2}
\end{array}\right)\left(U^{\left(k_{1}, j_{2}\right)}-\theta\right) \\
& +d\left(k_{2}, k_{2}\right)\left(\begin{array}{c}
n_{2} \\
k_{2}
\end{array}\right) \sum_{j_{1}=1}^{k_{1}-2} d\left(k_{1}, j_{1}\right)\left(\begin{array}{c}
n_{1} \\
j_{1}
\end{array}\right)\left(U_{n_{1}, n_{2}}^{\left(j_{1}, k_{2}\right)}-\theta\right) \\
& +\sum_{j_{1}=1}^{k_{1}-1} \sum_{j_{2}=1}^{k_{2}-1} d\left(k_{1}, j_{1}\right) d\left(k_{2}, j_{2}\right) \\
& \left.\times\left(\begin{array}{c}
n_{1} \\
j_{1}
\end{array}\right)\left(\begin{array}{c}
n_{2} \\
j_{2}
\end{array}\right)\left(U_{n_{1}, n_{2}}^{\left(j_{1}, j_{2}\right)}-\theta\right)\right]
\end{aligned}
$$

We evaluate $I_{n_{1}, n_{2}}^{(1)}, I_{n_{1}, n_{2}}^{(2)}, I_{n_{1}, n_{2}}^{(3)}, b_{n_{1}, n_{2}}$ and $R_{n_{1}, n_{2}}^{*}$ as the followings (i), (ii), (iii) and (iv).

(i) From (3.1), we have

$$
I_{n_{1}, n_{2}}^{(1)}=\frac{d\left(k_{1}, k_{1}\right)}{D\left(n_{1}, k_{1}\right)}\left(\begin{array}{l}
n_{1} \\
k_{1}
\end{array}\right) \frac{d\left(k_{2}, k_{2}\right)}{D\left(n_{2}, k_{2}\right)}\left(\begin{array}{l}
n_{2} \\
k_{2}
\end{array}\right)\left(U_{n_{1}, n_{2}}-\theta\right)
$$




$$
\begin{aligned}
& =\left(1-\frac{\delta_{k_{1}}}{n_{1}}-\frac{\delta_{k_{2}}}{n_{2}}+O\left(\frac{1}{N^{2}}\right)\right)\left(U_{n_{1}, n_{2}}-\theta\right) \\
& =\left(U_{n_{1}, n_{2}}-\theta\right)+R_{n_{1}, n_{2}}^{* *}
\end{aligned}
$$

where $E\left(R_{n_{1}, n_{2}}^{* *}\right)^{2}=O\left(N^{-3}\right)$ because of $\operatorname{Var}\left[U_{n_{1}, n_{2}}\right]=O\left(N^{-1}\right)$.

(ii) From (3.1) and (3.2), we have

$$
\begin{aligned}
I_{n_{1}, n_{2}}^{(2)} & =\frac{d\left(k_{1}, k_{1}\right)}{D\left(n_{1}, k_{1}\right)}\left(\begin{array}{c}
n_{1} \\
k_{1}
\end{array}\right) \frac{d\left(k_{2}, k_{2}-1\right)}{D\left(n_{2}, k_{2}\right)}\left(\begin{array}{c}
n_{2} \\
k_{2}-1
\end{array}\right)\left(U_{n_{1}, n_{2}}^{\left(k_{1}, k_{2}-1\right)}-\theta^{\left(k_{1}, k_{2}-1\right)}\right) \\
& =\left(1-\frac{\delta_{k_{1}}}{n_{1}}+O\left(\frac{1}{N}\right)\right) \frac{\delta_{k_{2}}}{n_{2}}\left(U_{n_{1}, n_{2}}^{\left(k_{1}, k_{2}-1\right)}-\theta^{\left(k_{1}, k_{2}-1\right)}\right) .
\end{aligned}
$$

By (3.5), we have

$$
E\left[I_{n_{1}, n_{2}}^{(2)}\right]^{2}=O\left(N^{-3}\right)
$$

Similarly,

$$
E\left[I_{n_{1}, n_{2}}^{(3)}\right]^{2}=O\left(N^{-3}\right) .
$$

(iii) From (3.1) and (3.2), we have

$$
\begin{aligned}
b_{n_{1}, n_{2}}= & \left(1-\frac{\delta_{k_{1}}}{n_{1}}+O\left(\frac{1}{N}\right)\right) \frac{\delta_{k_{2}}}{n_{2}}\left(\theta^{\left(k_{1}, k_{2}-1\right)}-\theta\right) \\
& +\left(1-\frac{\delta_{k_{2}}}{n_{2}}+O\left(\frac{1}{N}\right)\right) \frac{\delta_{k_{1}}}{n_{1}}\left(\theta^{\left(k_{1}-1, k_{2}\right)}-\theta\right) \\
= & \frac{\delta_{k_{1}}}{n_{1}}\left(\theta^{\left(k_{1}-1, k_{2}\right)}-\theta\right)+\frac{\delta_{k_{2}}}{n_{2}}\left(\theta^{\left(k_{1}, k_{2}-1\right)}-\theta\right)+O\left(N^{-2}\right) .
\end{aligned}
$$

Thus we get

$$
b_{n_{1}, n_{2}}=\frac{1}{N} b^{(0)}+o\left(N^{-1}\right) .
$$

(iv) Since $\operatorname{Var}\left[U_{n_{1}, n_{2}}^{\left(j_{1}, j_{2}\right)}\right]=O\left(N^{-1}\right)$ for $j_{1}, j_{2} \geq 1$ and $[d(k, j) / D(n, k)]\left(\begin{array}{c}n \\ j\end{array}\right)=$ $O\left(n^{-2}\right)(j=1, \ldots, k-2)$, we have

$$
E\left(R_{n_{1}, n_{2}}^{*}\right)^{2}=O\left(N^{-4}\right) \text {. }
$$

Applying (3.9), (3.10), (3.11), (3.12) and (3.13) to (3.8), we get (3.6).

\section{Edgeworth expansion}

For the two-sample U-statistic $U_{n_{1}, n_{2}}, \sqrt{N}\left(U_{n_{1}, n_{2}}-\theta\right)$ converges to Normal distribution $N\left(0, \sigma^{2}\right)$ as $N$ tends to $\infty$ (see, for example, Lee (1990), p. 141, and Randles and Wolfe (1979), p. 92). Therefore by (3.6), $\sqrt{N}\left(Y_{n_{1}, n_{2}}-\theta\right)$ converges to the same Normal distribution. To see the difference between asymptotic distributions of these two statistics, we shall derive the Edgeworth expansion of the statistic $Y_{n_{1}, n_{2}}$. We put as follows.

$$
\begin{aligned}
& \psi_{1,0}\left(x_{1}\right)=E\left[h\left(x_{1}, X_{2}, \ldots, X_{k_{1}} ; Y_{1}, \ldots, Y_{k_{2}}\right)\right], \\
& \psi_{0,1}\left(y_{1}\right)=E\left[h\left(X_{1}, X_{2}, \ldots, X_{k_{1}} ; y_{1}, Y_{2}, \ldots, Y_{k_{2}}\right)\right],
\end{aligned}
$$




$$
\begin{aligned}
& \psi_{2,0}\left(x_{1}, x_{2}\right)=E\left[h\left(x_{1}, x_{2}, X_{3}, \ldots, X_{k_{1}} ; Y_{1}, \ldots, Y_{k_{2}}\right)\right], \\
& \psi_{0,2}\left(y_{1}, y_{2}\right)=E\left[h\left(X_{1}, X_{2}, \ldots, X_{k_{1}} ; y_{1}, y_{2}, Y_{3}, \ldots, Y_{k_{2}}\right)\right], \\
& \psi_{1,1}\left(x_{1} ; y_{1}\right)=E\left[h\left(x_{1}, X_{2}, \ldots, X_{k_{1}} ; y_{1}, Y_{2}, \ldots, Y_{k_{2}}\right)\right] \\
& h^{(1,0)}\left(x_{1}\right)=\psi_{1,0}\left(x_{1}\right)-\theta, \quad h^{(0,1)}\left(y_{1}\right)=\psi_{0,1}\left(y_{1}\right)-\theta, \\
& h^{(2,0)}\left(x_{1}, x_{2}\right)=\psi_{2,0}\left(x_{1}, x_{2}\right)-\psi_{1,0}\left(x_{1}\right)-\psi_{1,0}\left(x_{2}\right)+\theta \\
& h^{(0,2)}\left(y_{1}, y_{2}\right)=\psi_{2,0}\left(x_{1}, x_{2}\right)-\psi_{0,1}\left(y_{1}\right)-\psi_{0,1}\left(y_{2}\right)+\theta \\
& h^{(1,1)}\left(x_{1} ; y_{1}\right)=\psi_{1,1}\left(x_{1} ; y_{1}\right)-\psi_{1,0}\left(x_{1}\right)-\psi_{0,1}\left(y_{1}\right)+\theta \\
& \delta_{1,0}^{2}=\operatorname{Var}\left(h^{(1,0)}\left(X_{1}\right)\right)=E\left[\psi_{1,0}\left(X_{1}\right)-\theta\right]^{2}, \\
& \delta_{0,1}^{2}=\operatorname{Var}\left(h^{(0,1)}\left(Y_{1}\right)\right)=E\left[\psi_{0,1}\left(Y_{1}\right)-\theta\right]^{2} .
\end{aligned}
$$

In this paper, we assume that

$$
\delta_{1,0}^{2}>0 \quad \text { and } \quad \delta_{0,1}^{2}>0 .
$$

That is, we assume that the kernel $h$ is non-degenerate. Next, we put

$$
\sigma_{N}^{2}=\operatorname{Var}\left[U_{n_{1}, n_{2}}\right]=E\left[\left(U_{n_{1}, n_{2}}-\theta\right)^{2}\right]
$$

and

$$
\sigma_{N}^{* 2}=\frac{k_{1}^{2}}{n_{1}} \delta_{1,0}^{2}+\frac{k_{2}^{2}}{n_{2}} \delta_{0,1}^{2} .
$$

Then, we have the relation

$$
\sigma_{N}^{2}=\sigma_{N}^{* 2}+O\left(N^{-2}\right)
$$

Furthermore, we put

$$
\sigma^{2}=\frac{k_{1}^{2}}{p} \delta_{1,0}^{2}+\frac{k_{2}^{2}}{1-p} \delta_{0,1}^{2}
$$

and

$$
\begin{aligned}
\eta_{2, N}=\frac{1}{\sigma_{N}^{* 3}}\{ & \frac{k_{1}^{3}}{n_{1}^{2}} E\left[\left(h^{(1,0)}\left(X_{1}\right)\right)^{3}\right]+\frac{k_{2}^{3}}{n_{2}^{2}} E\left[\left(h^{(0,1)}\left(Y_{1}\right)\right)^{3}\right] \\
& +\frac{6 k_{1}^{2} k_{2}^{2}}{n_{1} n_{2}} E\left[h^{(1,0)}\left(X_{1}\right) h^{(0,1)}\left(Y_{1}\right) h^{(1,1)}\left(X_{1} ; Y_{1}\right)\right] \\
& +\frac{3 k_{1}^{3}\left(k_{1}-1\right)}{n_{1}^{2}} E\left[h^{(1,0)}\left(X_{1}\right) h^{(1,0)}\left(X_{2}\right) h^{(2,0)}\left(X_{1}, X_{2}\right)\right] \\
& \left.+\frac{3 k_{2}^{3}\left(k_{2}-1\right)}{n_{2}^{2}} E\left[h^{(0,1)}\left(Y_{1}\right) h^{(0,1)}\left(Y_{2}\right) h^{(0,2)}\left(Y_{1}, Y_{2}\right)\right]\right\}
\end{aligned}
$$

The right-hand side of $\eta_{2, N}$ is due to Maesono (1985). The last three expectations on the right-hand side are rewritten as follows:

$$
\begin{aligned}
& E\left[h^{(1,0)}\left(X_{1}\right) h^{(0,1)}\left(Y_{1}\right) h^{(1,1)}\left(X_{1} ; Y_{1}\right)\right] \\
& \quad=E\left[\psi_{1,0}\left(X_{1}\right) \psi_{0,1}\left(Y_{1}\right) \psi_{1,1}\left(X_{1} ; Y_{1}\right)\right]-\theta\left(\delta_{1,0}^{2}+\delta_{0,1}^{2}\right)-\theta^{3},
\end{aligned}
$$




$$
\begin{aligned}
& E\left[h^{(1,0)}\left(X_{1}\right) h^{(1,0)}\left(X_{2}\right) h^{(2,0)}\left(X_{1}, X_{2}\right)\right] \\
& \quad=E\left[\psi_{1,0}\left(X_{1}\right) \psi_{1,0}\left(X_{2}\right) \psi_{2,0}\left(X_{1}, X_{2}\right)\right]-\theta \delta_{1,0}^{2}-\theta^{3}, \\
& E\left[h^{(0,1)}\left(Y_{1}\right) h^{(0,1)}\left(Y_{2}\right) h^{(0,2)}\left(Y_{1}, Y_{2}\right)\right] \\
& \quad=E\left[\psi_{0,1}\left(Y_{1}\right) \psi_{0,1}\left(Y_{2}\right) \psi_{0,2}\left(Y_{1}, Y_{2}\right)\right]-\theta \delta_{0,1}^{2}-\theta^{3} .
\end{aligned}
$$

We put

$$
Q(x)=\Phi(x)+\eta_{2, N}\left(1-x^{2}\right) \phi(x) .
$$

Let $\varphi_{1}$ and $\varphi_{2}$ be the characteristic functions of the random variables $h^{(1,0)}\left(X_{1}\right)$ and $h^{(0,1)}\left(Y_{1}\right)$, respectively. The following is the Edgeworth expansion of the two-sample U-statistic $U_{n_{1}, n_{2}}$ by Koroljuk and Borovskich (1994).

Lemma 4.1. (Koroljuk and Borovskich (1994), Theorem 6.3.2) Suppose that the Cramer condition

$$
\lim _{|t| \rightarrow \infty} \sup \left|\varphi_{j}(t)\right|<1, \quad j=1,2
$$

and the moment condition

$$
E\left[\left|h\left(X_{1}, \ldots, X_{k_{1}} ; Y_{1}, \ldots, Y_{k_{2}}\right)\right|^{3}\right]<\infty
$$

is satisfied. Then

$$
\sup _{x}\left|P\left(\sigma_{N}^{-1}\left(U_{n_{1}, n_{2}}-\theta\right) \leq x\right)-Q(x)\right|=O\left(N^{-3 / 5}\right)
$$

as $N \rightarrow \infty$.

Before giving the Edgeworth expansion of the statistic $Y_{n_{1}, n_{2}}$, we show the relation between the two statistics $U_{n_{1}, n_{2}}$ and $Y_{n_{1}, n_{2}}$.

Proposition 4.2. Suppose that $w(1, \ldots, 1 ; k)>0$ and (3.4) is satisfied. Then

$$
\begin{aligned}
\sup _{x} \mid & P\left(\sigma_{N}^{*-1}\left(Y_{n_{1}, n_{2}}-\theta\right) \leq x\right)-P\left(\sigma_{N}^{-1}\left(U_{n_{1}, n_{2}}-\theta\right)+\frac{1}{\sqrt{N} \sigma} b^{(0)} \leq x\right) \mid \\
& =o\left(N^{-1 / 2}\right) .
\end{aligned}
$$

Proof. From (3.6), we have

$$
\sigma_{N}^{*-1}\left(Y_{n_{1}, n_{2}}-\theta\right)=\sigma_{N}^{*-1}\left(U_{n_{1}, n_{2}}-\theta\right)+\frac{1}{N \sigma_{N}^{*}} b^{(0)}+R_{n_{1}, n_{2}}^{* * *}
$$

where $E\left[\left|R_{n_{1}, n_{2}}^{* * *}\right|\right]=o\left(N^{-1 / 2}\right)$.

Thus,

$$
\begin{aligned}
\sup _{x} \mid & P\left(\sigma_{N}^{*-1}\left(Y_{n_{1}, n_{2}}-\theta\right) \leq x\right)-P\left(\sigma_{N}^{*-1}\left(U_{n_{1}, n_{2}}-\theta\right)+\frac{1}{N \sigma_{N}^{*}} b^{(0)} \leq x\right) \mid \\
& =o\left(N^{-1 / 2}\right),
\end{aligned}
$$


where we use the relation

$$
\sup _{x}|P(W+\Delta \leq x)-P(W \leq x)| \leq 4(E|W \Delta|+E|\Delta|)
$$

for any random variables $W$ and $\Delta$ (see Shorack (2000), p. 261).

Since

$$
E\left[\left|\sigma_{N}^{*-1}-\sigma_{N}^{-1}\right| \cdot\left|U_{n_{1}, n_{2}}-\theta\right|\right]=O\left(N^{-1}\right),
$$

using the relation (4.6), we have

$$
\begin{aligned}
\sup _{x} \mid P\left(\sigma_{N}^{*-1}\left(U_{n_{1}, n_{2}}-\theta\right)+\frac{1}{N \sigma_{N}^{*}} b^{(0)} \leq x\right) & \\
& -P\left(\sigma_{N}^{-1}\left(U_{n_{1}, n_{2}}-\theta\right)+\frac{1}{N \sigma_{N}^{*}} b^{(0)} \leq x\right) \mid \\
= & O\left(N^{-1}\right) .
\end{aligned}
$$

Since

$$
\frac{1}{N \sigma_{N}^{*}}-\frac{1}{\sqrt{N} \sigma}=o\left(N^{-1 / 2}\right)
$$

we have

$$
\begin{aligned}
\sup _{x} \mid P\left(\sigma_{N}^{-1}\left(U_{n_{1}, n_{2}}-\theta\right)+\frac{1}{N \sigma_{N}^{*}} b^{(0)} \leq x\right) \\
\quad-P\left(\sigma_{N}^{-1}\left(U_{n_{1}, n_{2}}-\theta\right)+\frac{1}{\sqrt{N} \sigma} b^{(0)} \leq x\right) \mid \\
=o\left(N^{-1 / 2}\right) .
\end{aligned}
$$

Thus by (4.5), (4.7) and (4.8), we get (4.4).

We put

$$
\begin{aligned}
Q^{*}(x) & =Q(x)+\frac{b^{(0)}}{\sqrt{N} \sigma}\left[-\frac{1}{2} \phi(x)+x\left(1-x^{2}\right) \phi(x)\right] \\
& =\Phi(x)-\frac{b^{(0)}}{2 \sqrt{N} \sigma} \phi(x)+\eta_{2, N}\left(1-x^{2}\right) \phi(x)+\frac{b^{(0)}}{\sqrt{N} \sigma} x\left(1-x^{2}\right) \phi(x) .
\end{aligned}
$$

The Edgeworth expansion (4.3) of $U_{n_{1}, n_{2}}$ is derived by standardising with its standard deviation $\sigma_{N}$. Since $\sigma_{N}$ is not the standard deviation of the statistic $Y_{n_{1}, n_{2}}$, we shall standardise $Y_{n_{1}, n_{2}}$ by using its asymptotic standard deviation $\sigma_{N}^{*}$ and obtain the Edgeworth expansion of $Y_{n_{1}, n_{2}}$ with the remainder term $o\left(N^{-1 / 2}\right)$ as follows.

TheOrem 4.3. Suppose that $w(1, \ldots, 1 ; k)>0$, and $(3.4),(4.1)$ and $(4.2)$ are satisfied. Then

$$
\sup _{x}\left|P\left(\sigma_{N}^{*-1}\left(Y_{n_{1}, n_{2}}-\theta\right) \leq x\right)-Q^{*}(x)\right|=o\left(N^{-1 / 2}\right) .
$$


Proof. From (4.3), we have

$$
\sup _{x}\left|P\left(\sigma_{N}^{-1}\left(U_{n_{1}, n_{2}}-\theta\right) \leq x-\frac{1}{\sqrt{N} \sigma} b^{(0)}\right)-Q\left(x-\frac{1}{\sqrt{N} \sigma} b^{(0)}\right)\right|=O\left(N^{-3 / 5}\right) \text {. }
$$

Thus by this relation and (4.4), we get

$$
\sup _{x}\left|P\left(\sigma_{N}^{*-1}\left(Y_{n_{1}, n_{2}}-\theta\right) \leq x\right)-Q\left(x-\frac{1}{\sqrt{N} \sigma} b^{(0)}\right)\right|=o\left(N^{-1 / 2}\right),
$$

where

$$
\begin{aligned}
Q\left(x-\frac{b^{(0)}}{\sqrt{N} \sigma}\right)= & \Phi\left(x-\frac{1}{\sqrt{N} \sigma} b^{(0)}\right) \\
& +\eta_{2, N}\left[1-\left(x-\frac{1}{\sqrt{N} \sigma} b^{(0)}\right)^{2}\right] \phi\left(x-\frac{1}{\sqrt{N} \sigma} b^{(0)}\right) \\
= & Q^{*}(x)+O\left(N^{-1}\right) .
\end{aligned}
$$

Thus by (4.11) and (4.12), we get (4.10).

COROLlary 4.4. The difference between the Edgeworth expansions of the two-sample U-statistic $U_{n_{1}, n_{2}}$ and the statistic $Y_{n_{1}, n_{2}}$ is given by

$$
\frac{b^{(0)}}{\sqrt{N} \sigma}\left[-\frac{1}{2} \phi(x)+x\left(1-x^{2}\right) \phi(x)\right] \text {. }
$$

\section{Examples}

The asymptotic expansion of $\left(Y_{n_{1}, n_{2}}-\theta\right) / \sigma_{N}, Q^{*}(x)$, depends on $b^{(0)}$ and $\eta_{2, N}$. For 4 kernels we shall give the values of $b^{(0)}$ and $\eta_{2, N}$ about $V_{n_{1}, n_{2}}, S_{n_{1}, n_{2}}$ and $B_{n_{1}, n_{2}}$, based on some special distributions.

(i) We consider the kernel

$$
h\left(x_{1}, \ldots, x_{r} ; y_{1}, \ldots, y_{r}\right)=x_{1} \cdots x_{r}-y_{1} \cdots y_{r}, \quad r=2,3, \ldots
$$

which gives the parameter $\theta=\mu^{r}-\nu^{r}$, where $\mu=E\left(X_{1}\right)$ and $\nu=E\left(Y_{1}\right)$. We assume that $X$ and $Y$ are symmetric about $\mu$ and $\nu$, respectively. Then we have

$$
\theta^{(r-1, r)}=E\left(X_{1}^{2}\right) \cdot \mu^{r-2}-\nu^{r}, \quad \theta^{(r, r-1)}=\mu^{r}-E\left(Y_{1}^{2}\right) \cdot \nu^{r-2},
$$

and

$$
b^{(0)}=\delta_{r}\left[\frac{1}{p} \mu^{r-2} \operatorname{Var}\left(X_{1}\right)-\frac{1}{1-p} \nu^{r-2} \operatorname{Var}\left(Y_{1}\right)\right]
$$

where $\delta_{r}=r(r-1) / 2$ for $V_{n_{1}, n_{2}}$ and $S_{n_{1}, n_{2}}$, and $\delta_{r}=r(r-1)$ for $B_{n_{1}, n_{2}}$.

Next we evaluate $\eta_{2, N}$. We have

$$
\begin{aligned}
& h^{(1,0)}\left(x_{1}\right)=\mu^{r-1}\left(x_{1}-\mu\right), \quad h^{(0,1)}\left(y_{1}\right)=-\nu^{r-1}\left(y_{1}-\nu\right), \\
& h^{(2,0)}\left(x_{1}, x_{2}\right)=\mu^{r-2}\left(x_{1}-\mu\right)\left(x_{2}-\mu\right), \\
& h^{(0,2)}\left(y_{1}, y_{2}\right)=-\nu^{r-2}\left(y_{1}-\mu\right)\left(y_{2}-\mu\right), \quad \text { and } \quad h^{(1,1)}\left(x_{1} ; y_{1}\right)=0 .
\end{aligned}
$$


Thus we get

$$
\delta_{1,0}^{2}=\mu^{2 r-2} \operatorname{Var}\left(X_{1}\right), \quad \delta_{0,1}^{2}=\nu^{2 r-2} \operatorname{Var}\left(Y_{1}\right)
$$

and

$$
\sigma_{N}^{* 2}=r^{2}\left(\frac{\mu^{2 r-2} \operatorname{Var}\left(X_{1}\right)}{n_{1}}+\frac{\nu^{2 r-2} \operatorname{Var}\left(Y_{1}\right)}{n_{2}}\right) .
$$

Therefore, we have

$$
\begin{aligned}
\eta_{2, N}= & 3(r-1)\left(\frac{\mu^{3 r-4}\left[\operatorname{Var}\left(X_{1}\right)\right]^{2}}{n_{1}^{2}}-\frac{\nu^{3 r-4}\left[\operatorname{Var}\left(Y_{1}\right)\right]^{2}}{n_{2}^{2}}\right) \\
& \times\left(\frac{\mu^{2 r-2} \operatorname{Var}\left(X_{1}\right)}{n_{1}}+\frac{\nu^{2 r-2} \operatorname{Var}\left(Y_{1}\right)}{n_{2}}\right)^{-3 / 2} .
\end{aligned}
$$

(ii) We consider the kernel given by

$$
h\left(x_{1}, x_{2} ; y_{1}, y_{2}\right)= \begin{cases}1 & \left(x_{1}, x_{2}<y_{1}, y_{2} \text { or } x_{1}, x_{2}>y_{1}, y_{2}\right) \\ 0 & \text { otherwise. }\end{cases}
$$

The corresponding parameter $\theta=E h\left(X_{1}, X_{2} ; Y_{1}, Y_{2}\right)$ is equal to $2 \Delta+1 / 3$, where $\Delta$ is given by (iii) of Section 1 . We consider the uniform distributions $U(0,1)$ and $U(1 / 2,3 / 2)$ as $F$ and $G$, respectively. Then we have

$$
\theta=\frac{2}{3}, \quad \theta^{(1,2)}=\frac{5}{6} \quad \text { and } \quad \theta^{(2,1)}=\frac{5}{6} .
$$

Thus we get

$$
b^{(0)}=\frac{\delta_{2}}{6 p(1-p)},
$$

where $\delta_{2}=1$ for $V_{n_{1}, n_{2}}$ and $S_{n_{1}, n_{2}}$, and $\delta_{2}=2$ for $B_{n_{1}, n_{2}}$.

For $0<x_{1}, x_{2}<1$ and $1 / 2<y_{1}, y_{2}<3 / 2$, we have

$$
\begin{aligned}
& \psi_{1,0}\left(x_{1}\right)= \begin{cases}\frac{19}{24} & \left(0<x_{1}<1 / 2\right) \\
-x_{1}+\frac{31}{24} & \left(1 / 2 \leq x_{1}<1\right),\end{cases} \\
& \psi_{0,1}\left(y_{1}\right)= \begin{cases}y_{1}-\frac{5}{24} & \left(1 / 2<y_{1}<1\right) \\
\frac{19}{24} & \left(1 \leq y_{1}<3 / 2\right),\end{cases} \\
& \psi_{2,0}\left(x_{1}, x_{2}\right)=\left[\frac{3}{2}-\max \left(x_{1}, x_{2}\right)\right]^{2} I_{(0.5,1)}\left(\max \left(x_{1}, x_{2}\right)\right)+I_{(0,0.5)}\left(\max \left(x_{1}, x_{2}\right)\right) \\
& +\left[\min \left(x_{1}, x_{2}\right)-\frac{1}{2}\right]^{2} I_{(0.5,1)}\left(\min \left(x_{1}, x_{2}\right)\right) \\
& \psi_{0,2}\left(y_{1}, y_{2}\right)=\left[\min \left(y_{1}, y_{2}\right)\right]^{2} I_{(0.5,1)}\left(\min \left(y_{1}, y_{2}\right)\right)+I_{(1,1.5)}\left(\min \left(y_{1}, y_{2}\right)\right) \\
& +\left[1-\max \left(y_{1}, y_{2}\right)\right]^{2} I_{(0.5,1)}\left(\max \left(y_{1}, y_{2}\right)\right) \text {, }
\end{aligned}
$$


and

$$
\psi_{1,1}\left(x_{1} ; y_{1}\right)= \begin{cases}\frac{7}{8} & \left(0<x_{1}<1 / 2, y_{1}>1\right) \\ -\frac{1}{8}+\frac{3}{2} y_{1}-\frac{1}{2} y_{1}^{2} & \left(0<x_{1}<1 / 2, y_{1} \leq 1\right) \\ 1-\frac{1}{2} x_{1}^{2} & \left(1 / 2<x_{1}<1,1 \leq y_{1}<3 / 2\right) \\ \frac{3}{2} y_{1}-\frac{1}{2}\left(x_{1}^{2}+y_{1}^{2}\right) & \left(1 / 2 \leq y_{1}<1,1 / 2 \leq x_{1}<1, x_{1}<y_{1}\right) \\ -\frac{1}{2}+x_{1}+\frac{1}{2} y_{1}-\frac{1}{2}\left(x_{1}^{2}+y_{1}^{2}\right) & \left(1 / 2 \leq y_{1}<1,1 / 2 \leq x_{1}<1, x_{1}>y_{1}\right) .\end{cases}
$$

Thus by using Mathematica to compute the integrals we get

$$
\begin{aligned}
\delta_{1,0}^{2} & =E\left[\psi_{1,0}\left(X_{1}\right)-\theta\right]^{2}=\frac{5}{192}, \quad \delta_{0,1}^{2}=E\left[\psi_{0,1}\left(Y_{1}\right)-\theta\right]^{2}=\frac{5}{192}, \\
\sigma_{N}^{* 2} & =\frac{10}{81}\left(\frac{1}{n_{1}}+\frac{1}{n_{2}}\right)
\end{aligned}
$$

and

$$
\begin{aligned}
& E\left[\left(h^{(1,0)}\left(X_{1}\right)\right)^{3}\right]=-\frac{1}{256}, \quad E\left[\left(h^{(0,1)}\left(Y_{1}\right)\right)^{3}\right]=-\frac{1}{256}, \\
& E\left[h^{(1,0)}\left(X_{1}\right) h^{(0,1)}\left(Y_{1}\right) h^{(1,1)}\left(X_{1} ; Y_{1}\right)\right]=-\frac{1}{960}, \\
& E\left[h^{(1,0)}\left(X_{1}\right) h^{(1,0)}\left(X_{2}\right) h^{(2,0)}\left(X_{1}, X_{2}\right)\right]=\frac{13}{2560}, \\
& E\left[h^{(0,1)}\left(Y_{1}\right) h^{(0,1)}\left(Y_{2}\right) h^{(0,2)}\left(Y_{1}, Y_{2}\right)\right]=\frac{107}{20480} .
\end{aligned}
$$

Therefore we have

$$
\eta_{2, N}=\frac{729 \sqrt{10}}{256000}\left(\frac{232}{n_{1}^{2}}+\frac{241}{n_{2}^{2}}-\frac{256}{n_{1} n_{2}}\right)\left(\frac{1}{n_{1}}+\frac{1}{n_{2}}\right)^{-3 / 2} .
$$

(iii) The kernel given by (iv) of Section 1 is considered. We have $\theta^{(1,2)}=1$ and $\theta^{(2,1)}=0$. We consider the uniform ditributions $U(0,1)$ and $U(1 / 4,3 / 4)$ as $F$ and $G$, respectively. Then we have $\theta=7 / 24$ and

$$
b^{(0)}=\frac{\delta_{2}}{p(1-p)}\left(\frac{17}{24}-p\right) .
$$

Next we evaluate $\eta_{2, N}$. For $0<x_{1}, x_{2}<1$ and $1 / 4<y_{1}, y_{2}<3 / 4$ we have

$$
\begin{aligned}
& \psi_{1,0}\left(x_{1}\right)=\frac{1}{3}-\frac{4}{3}\left(x_{1}-\frac{1}{2}\right)^{3}, \\
& \psi_{0,1}\left(y_{1}\right)=2\left\{\left(y_{1}-\frac{1}{4}\right)^{2}+\left(\frac{3}{4}-y_{1}\right)^{2}\right\}+\frac{2}{3}\left\{\left(y_{1}-\frac{1}{4}\right)^{3}-\left(\frac{3}{4}-y_{1}\right)^{3}\right\}, \\
& \psi_{2,0}\left(x_{1}, x_{2}\right)= \begin{cases}\left(1-2\left|x_{1}-x_{2}\right|\right)^{2} & \left(2\left|x_{1}-x_{2}\right|<1\right) \\
0 & \left(2\left|x_{1}-x_{2}\right| \geq 1\right),\end{cases}
\end{aligned}
$$


and

$$
\psi_{0,2}\left(y_{1}, y_{2}\right)=2\left|y_{1}-y_{2}\right|-\left(y_{1}-y_{2}\right)^{2} .
$$

Futhermore, for $0<x_{1}<1 / 2$ and $y_{1}+x_{1}<3 / 4$

$$
\psi_{1,1}\left(x_{1} ; y_{1}\right)= \begin{cases}\left(x_{1}+y_{1}-\frac{1}{4}\right)^{2}+\left(x_{1}-y_{1}+\frac{3}{4}\right)^{2}-4 x_{1}^{2} & \left(y_{1}-x_{1}>1 / 4\right) \\ \left(x_{1}-y_{1}+\frac{3}{4}\right)^{2}+2\left(y_{1}-\frac{1}{4}\right)^{2}-2 x_{1}^{2} & \left(y_{1}-x_{1} \leq 1 / 4\right) .\end{cases}
$$

For $0<x_{1}<1 / 2$ and $y_{1}+x_{1}>3 / 4$

$$
\psi_{1,1}\left(x_{1} ; y_{1}\right)= \begin{cases}\left(x_{1}+y_{1}-\frac{1}{4}\right)^{2}-2 x_{1}^{2}+2\left(y_{1}-\frac{3}{4}\right)^{2} & \left(y_{1}-x_{1}>1 / 4\right) \\ 2\left\{\left(y_{1}-\frac{1}{4}\right)^{2}+\left(y_{1}-\frac{3}{4}\right)^{2}\right\} & \left(y_{1}-x_{1} \leq 1 / 4\right) .\end{cases}
$$

For $1 / 2<x_{1}<1$ and $y_{1}+x_{1}-1<1 / 4$,

$$
\psi_{1,1}\left(x_{1} ; y_{1}\right)= \begin{cases}2\left\{\left(y_{1}-\frac{1}{4}\right)^{2}+\left(y_{1}-\frac{3}{4}\right)^{2}\right\} & \left(y_{1}-x_{1}+1>3 / 4\right) \\ 2\left(y_{1}-\frac{1}{4}\right)^{2}+\left(\frac{5}{4}-x_{1}-y_{1}\right)^{2}-2\left(1-x_{1}\right)^{2} & \left(y_{1}-x_{1}+1 \leq 3 / 4\right) .\end{cases}
$$

For $1 / 2<x_{1}<1$ and $y_{1}+x_{1}-1>1 / 4$,

$$
\psi_{1,1}\left(x_{1} ; y_{1}\right)=\left\{\begin{array}{c}
\left(\frac{3}{4}-x_{1}+y_{1}\right)^{2}+\left(\frac{7}{4}-x_{1}-y_{1}\right)^{2}-4\left(1-x_{1}\right)^{2} \\
\left(y_{1}-x_{1}+1<3 / 4\right) \\
\left(\frac{3}{4}-x_{1}+y_{1}\right)^{2}-2\left(1-x_{1}\right)^{2}+2\left(\frac{3}{4}-y_{1}\right)^{2} \\
\left(y_{1}-x_{1}+1>3 / 4\right) .
\end{array}\right.
$$

Thus by using Mathematica to compute the integrals we get

$$
\begin{aligned}
& \delta_{1,0}^{2}=E\left[\psi_{1,0}\left(X_{1}\right)-\theta\right]^{2}=\frac{23}{4032} \\
& \delta_{0,1}^{2}=E\left[\psi_{0,1}\left(Y_{1}\right)-\theta\right]^{2}=\frac{37}{4032} \\
& \sigma_{N}^{* 2}=\frac{1}{1008}\left(\frac{23}{n_{1}}+\frac{37}{n_{2}}\right)
\end{aligned}
$$

and

$$
E\left[\left(h^{(1,0)}\left(X_{1}\right)\right)^{3}\right]=\frac{55}{96768}, \quad E\left[\left(h^{(0,1)}\left(Y_{1}\right)\right)^{3}\right]=\frac{821}{483840},
$$




$$
\begin{aligned}
& E\left[h^{(1,0)}\left(X_{1}\right) h^{(0,1)}\left(Y_{1}\right) h^{(1,1)}\left(X_{1} ; Y_{1}\right)\right]=\frac{50531}{92897280} \\
& E\left[h^{(1,0)}\left(X_{1}\right) h^{(1,0)}\left(X_{2}\right) h^{(2,0)}\left(X_{1}, X_{2}\right)\right]=\frac{3569}{2419200} \\
& E\left[h^{(0,1)}\left(Y_{1}\right) h^{(0,1)}\left(Y_{2}\right) h^{(0,2)}\left(Y_{1}, Y_{2}\right)\right]=-\frac{1009}{1036800}
\end{aligned}
$$

Therefore we have

$$
\eta_{2, N}=\frac{\sqrt{7}}{400}\left(\frac{193312}{n_{1}^{2}}-\frac{47328}{n_{2}^{2}}+\frac{252655}{n_{1} n_{2}}\right)\left(\frac{23}{n_{1}}+\frac{37}{n_{2}}\right)^{-3 / 2} .
$$

(iv) We consider the kernel given by (v) of Section 1. We consider the uniform ditributions $U(0,1)$ and $U(1 / 2,3 / 2)$ as $F$ and $G$, respectively. Then we have

$$
\theta^{(1,2)}=\frac{5}{6}, \quad \theta^{(2,1)}=\frac{11}{12}, \quad \theta=\frac{23}{24},
$$

and

$$
b^{(0)}=-\frac{\delta_{2}(3-2 p)}{24 p(1-p)} .
$$

For $0<x_{1}, x_{2}<1$ and $1 / 2<y_{1}, y_{2}<3 / 2$,

$$
\begin{aligned}
& \psi_{1,0}\left(x_{1}\right)=1-\frac{1}{6} x_{1}^{3}, \quad \psi_{0,1}\left(y_{1}\right)=1-\frac{1}{6}\left(\frac{3}{2}-y_{1}\right)^{3}, \\
& \psi_{2,0}\left(x_{1}, x_{2}\right)= \begin{cases}1 & \left(0<x_{1}+x_{2} \leq 1\right) \\
1-\frac{1}{2}\left(x_{1}+x_{2}-1\right)^{2} & \left(1<x_{1}+x_{2}<2\right),\end{cases} \\
& \psi_{0,2}\left(y_{1}, y_{2}\right)= \begin{cases}1-\frac{1}{2}\left[2-\left(y_{1}+y_{2}\right)\right]^{2} & \left(1<y_{1}+y_{2}<2\right) \\
1 & \left(2 \leq y_{1}+y_{2}<3\right),\end{cases}
\end{aligned}
$$

and

$$
\psi_{1,1}\left(x_{1} ; y_{1}\right)= \begin{cases}\frac{7}{8}+\frac{1}{2}\left(y_{1}-x_{1}\right)-\frac{1}{2}\left(y_{1}-x_{1}\right)^{2} & \left(-\frac{1}{2}<y_{1}-x_{1}<\frac{1}{2}\right) \\ 1 & \left(\frac{1}{2} \leq y_{1}-x_{1}<\frac{3}{2}\right) .\end{cases}
$$

Thus by using Mathematica to compute the integrals we get

$$
\begin{array}{ll}
\delta_{1,0}^{2}=\frac{1}{448}, \quad \delta_{0,1}^{2}=\frac{1}{448}, & \sigma_{N}^{* 2}=\frac{1}{112}\left(\frac{1}{n_{1}}+\frac{1}{n_{2}}\right), \\
E\left[\left(h^{(1,0)}\left(X_{1}\right)\right)^{3}\right]=-\frac{1}{8960}, & E\left[\left(h^{(0,1)}\left(Y_{1}\right)\right)^{3}\right]=-\frac{1}{8960},
\end{array}
$$




$$
\begin{aligned}
& E\left[h^{(1,0)}\left(X_{1}\right) h^{(0,1)}\left(Y_{1}\right) h^{(1,1)}\left(X_{1} ; Y_{1}\right)\right]=-\frac{9727}{7257600}, \\
& E\left[h^{(1,0)}\left(X_{1}\right) h^{(1,0)}\left(X_{2}\right) h^{(2,0)}\left(X_{1}, X_{2}\right)\right]=-\frac{9727}{7257600}, \\
& E\left[h^{(0,1)}\left(Y_{1}\right) h^{(0,1)}\left(Y_{2}\right) h^{(0,2)}\left(Y_{1}, Y_{2}\right)\right]=-\frac{32531}{7257600} .
\end{aligned}
$$

Therefore we have

$$
\eta_{2, N}=-\frac{\sqrt{7}}{675}\left(\frac{9997}{n_{1}^{2}}+\frac{32801}{n_{2}^{2}}+\frac{38908}{n_{1} n_{2}}\right)\left(\frac{1}{n_{1}}+\frac{1}{n_{2}}\right)^{-3 / 2} .
$$

\section{Acknowledgements}

We would like to express our thanks to Prof. Maesono for the discussions on the related matters, and the two referees for their kind suggestions.

\section{REFERENCES}

Charalambides, Ch. A. and Singh, J. (1988). A review of the Stirling numbers, their generalizations and statistical applications, Commun. Statist.-Theory Meth., 17, 2533-2595.

Ferguson, T. S. (1973). A Bayesian analysis of some nonparametric problems, Ann. Statist., 1, 209-230.

Hollander, M. (1967). Asymptotic efficiency of two nonparametric competitors of Wilcoxon's two sample test, J. Amer. Statist. Assoc., 62, 939-949.

Koroljuk, V. S. and Borovskich, Yu. V. (1994). Theory of U-statistics, Kluwer Academic Publishers, Dordrecht.

Lee, A. J. (1990). U-statistics, Marcel Dekker, New York.

Lehmann, E. L. (1951). Consistency and unbiasedness of certain nonparametric tests, Ann. Math. Statist., 22, 165-179.

Maesono, Y. (1985). Edgeworth expansion for two-sample U-statistics, Rep. Fac. Sci. Kagoshima Univ., (Math., Phys. \& Chem.), No. 18, 35-43.

Nomachi, T., Kondo, M. and Yamato, H. (2002). Higher Order efficiency of linear combinations of U-statistics as estimators of estimable parameters, Scientiae Mathematicae Japonicae, 56, 95-106.

Randles, R. H. and Wolfe, D. A. (1979). Introduction to the Theory of Nonparametric Statistics, John Wiley, New York.

Shorack, G. R. (2000). Probability for Statisticians, Springer, New York.

Toda, K. and Yamato, H. (2001). Berry-Esseen bounds for some statistics including LB-statistic and V-statistic, J. Japan Statist. Soc., 31, 225-237.

Yamato, H. (1977). Relations between limiting Bayes estimates and U-statistics for estimable parameters, J. Japan Statist. Soc., 7, 57-66.

Yamato, H., Nomachi, T. and Toda, K. (2003). Edgeworth expansions of some statistics including the LB-statistic and V-statistic, J. Japan Statist. Soc., 33, 77-94. 